\title{
A GENERALIZATION OF THE NOTION OF ANGLE*
}

\author{
BY \\ GILBERT AMES BLISS
}

The tendency of recent writers has been to regard geometry as the science of a certain special kind of assemblage. $\dagger$ The elements of this assemblage, in deference to our intuitive notions called points, fall into subsets called lines. Among the lines and the points of any one line, such properties of intersection, congruence, order and continuity are presupposed as the particular geometer in question is pleased to prescribe, provided only that all the assumptions are mutually compatible. If it is desired that any two geometries satisfying the initial assumptions shall be essentially the same, the axioms must also form a so-called categorical system. $\ddagger$

The assemblage which forms the basis on which the usual Euclidean and nonEuclidean geometries are built up, is such that its elements can be put into one to one correspondence with the number ratios $x: y: z$, in which $(x, y, z)$ may have any real values different from $(0,0,0)$. Starting with assumptions which characterize this assemblage, HAMEL $\ddagger$ has constructed still more general geometries all sharing the property that straight lines are the shortest distances, and more recently STROMQuist $\S$ has discussed one in which circles are the shortest.

$\mathrm{H}_{A M E L}$, whose geometries are the most general in which straight lines are shortest, considers the points in his assemblage represented in the usual way on

\footnotetext{
* Presented to the Society at the Williamstown summer meeting, September 7, 1905. Received for publication October 10, 1905.

† Hilbert considers two related agsemblages, one of points and the other of lines; see his Foundations of Geometry, translated by E. J. Townsend.

$\ddagger$ I. e., a system such that the elements of any two assemblages satisfying it can be put into one to one correspondence in such a way that corresponding elements have similar properties; see Veblen, Transactions of the American Mathematical Society, vol. 5 (1904), p. 346. The arbitrariness in the choice of assumptions is well illustrated by the geometries in which there are only a finite number of points and lines; see the abstract of a paper by VEBLEN and Bussey, Bulletin of the American Mathematical Society, vol. 11 (1905), p. 480.

$\ddagger$ Über die Geometrieen in denen die Geraden die Kürzesten sind, Dissertation, Göttingen, 1901 ; Mathematisohe Annalen, vol. 57 (1903), p. 231.

\$ These Transactions, vol. 7 (1906), p. 175. An abstract of his paper may be found in the Bnlletin of the American Mathematical Society, vol. 9 (1903), p. 396.
} 
an $x y$-plane and the line at infinity. He then defines the length of a curve to be the value of an integral of the form

$$
I=\int f\left(x, y, y^{\prime}\right) d x,
$$

a generalization from the usual definition of length by the integral

$$
I=\int \sqrt{1+y^{\prime 2}} d x .
$$

But he omits to show the corresponding generalization of the notion of angle. It is the purpose of this paper to fill in this gap, and to show that the generalization of angle found agrees with the current definitions in the special cases of the non-Euclidean geometries and of geometry on a curved surface. The integral which defines the generalized angle for HAMEL's geometries turns out to be a specialization of an integral invariant connected with a more general problem of the calculus of variations.

In deriving the results of the paper the curves are considered in parametric representation, a form more satisfactory than the other from the geometrical standpoint, and a new form of the definite integral representing length is used which in itself is of interest to the student of the calculus of variations. Its introduction is especially desirable for the reason that the use of the ordinary Weierstrassian form of integral for curves in parametric representation presupposes HAMEL's weak monodromy axiom, * as will be explained in $\S 1$.

\section{§1. The length integral and its extremals.}

Suppose with Hamel that the points of the geometry to be studied are the $x y$-points of a plane and the line at infinity, that is, the elements of the geometry are to be in one to one correspondence with the number ratios $x: y: \dot{z}$, and the ratios with $z \neq 0$ are to be represented in the Cartesian plane. The others constitute the line at infinity. $\dagger$ A curve is then the totality of points defined in the usual way by two equations

$$
x=\phi(t), \quad y=\psi(t),
$$

and the length of an arc from $A\left(t=t_{0}\right)$ to $B\left(t=t_{1}>t_{0}\right)$ is defined to be the value of a definite integral

$$
l=\int_{t_{0}}^{t_{1}} f(x, y, \tau) \sqrt{{x^{\prime 2}+y^{\prime 2}}^{2}} d t .
$$

In this expression $\tau$ is the angle between the $x$-axis and the tangent to the curve and is defined by the equations

$$
\cos \tau=\frac{x^{\prime}}{\sqrt{x^{\prime 2}+y^{\prime 2}}}, \quad \sin \tau=\frac{y^{\prime}}{\sqrt{x^{\prime 2}+y^{\prime 2}}} .
$$

* Mathematische Annalen, vol. 57 (1903), p. 244.

$\dagger$ The methods given here apply only to the finite part of the plane. Foi points on the line at infinity or for curves having such points, a special discussion is necessary. 
The integral which WeIERSTRAss introduced into the calculus of variations * is

$$
I=\int F\left(x, y, x^{\prime}, y^{\prime}\right) d t,
$$

where the function $F$ is single valued for $x^{\prime}, y^{\prime}$ not both zero, and positively homogeneous of order one in $x^{\prime}$ and $y^{\prime}$. On account of the homogeneity it can be written in the form

$$
I=\int F(x, y, \cos \tau, \sin \tau) \sqrt{x^{\prime 2}+y^{\prime 2}} d t,
$$

which if used as a definition of length would mean that the function $f(x, y, \tau)$ in (2) would have the period $2 \pi$. No such assumption reed, however, be made on the function $f$, and it is desirable not to do so in order to avoid specializing the nature of the geometries which may be derived by HAMEL's method:

In order to carry through the methods of the following pages without going into too much detail with regard to initial hypotheses, it will be assumed that the functions $\phi$ and $\psi$ have continuous first and second derivatives, and that $f(x, y, \tau)$ has continuous partial derivatives of the first three orders for all values of $\tau$ and for values of $(x, y)$ in the neighborhood of the point $O\left(x_{0}, y_{0}\right)$ at which the generalization of angle is to be made. Furthermore $f\left(x_{0}, y_{0}, \tau\right)$ and $f-! \cdot f_{\tau \tau} \dagger$ for the same arguments are assumed to be greater than zero whatever $\tau$ may be. The last conditions will also be true for $(x, y)$ in a properly chosen neighborhood of the point $O$ provided that $\tau$ is restricted to some finite interval.

The differential equation which defines the curves of shortest length or extremals for the integral (2), is readily derivable by the method well known in the calculus of variations + . Suppose that the equations $(1)$ represent such an extremal joining $A\left(t=t_{0}\right)$ with $B\left(t=t_{1}\right)$, and let $\xi, \eta$ be two functions having continuous derivatives and vanishing at $t_{0}$ and $t_{1}$. Then the equations

$$
C_{a}: \quad \bar{r}=\phi(t)+\alpha \xi(t), \quad \bar{y}=\psi(t)+\alpha \eta(t),
$$

represent a set of curres each passing through $A$ and $B$ and reducing to the curve (1) when $\alpha=0$. The generalized length of the arc $A B$ of $C_{\alpha}$ has a value depending only upon $\alpha$, and is expressible in the form

$$
l(\alpha)=\int_{t_{0}}^{t_{1}} f(\bar{x}, y, \bar{\tau}) \sqrt{{\overline{x^{\prime}}}^{\prime 2}+\bar{y}^{\prime 2}} d t
$$

where $\bar{x}, \bar{y}, \bar{\tau}$ have the same meaning for $C_{a}$ which $x, y, \tau$ have for $C$. Then if

* See Bolza, Lectures on the Calculus of Variations, p. 117.

$\dagger$ Literal subscripts denote partial differentiation. The. expression $f+f_{\tau \tau}$ corresponds to the functions $f_{y^{\prime} y^{\prime}}$ and $F_{1}$ in other forms of the theory ; see BoLZA, loc. cit., pp. 47, 121 .

$\ddagger$ Bolza, loc. cit., p. 122 . 
the curve $C$ has the shortest length, the derivative $d l / d \alpha$ must vanish for $\alpha=0$. This derivative is

$$
\left[\begin{array}{l}
d l \\
\overline{d a}
\end{array}\right]_{a=0}=\int_{t_{0}}^{t_{1}}\left\{\left[f_{x} \xi+f_{y} \eta+f_{\tau} \frac{\partial \bar{\tau}}{\partial \alpha}\right] \sqrt{{x^{\prime 2}+y^{\prime 2}}^{2}}+f \frac{x^{\prime} \xi+y^{\prime} \eta}{\sqrt{x^{\prime 2}+y^{\prime 2}}}\right\} d t
$$

where the arguments of $f$ and its derivatives $f_{x}, f_{y}, f_{\tau}$ are $x, y, \tau$ from equations (1) and (3), and $\alpha$ has the value zero in $\partial \bar{\tau} / \partial \alpha$. The value of the last expression can be calculated from equations (3) in which $\bar{x}$ and $\bar{y}$ must be substituted for $x$ and $y$ :

$$
\left[\begin{array}{l}
\partial \bar{\tau} \\
\partial \alpha
\end{array}\right]_{a=0}=\frac{x^{\prime} \eta^{\prime}-y^{\prime} \xi^{\prime}}{x^{\prime 2}+y^{\prime 2}}
$$

After some simple alterations equation (4) takes the form

$\left[\begin{array}{l}d l \\ \bar{d} \alpha\end{array}\right]_{a=0}=\int_{s_{0}}^{s_{1}}\left\{f_{x} \xi+f_{y} \eta+\left(f \cos \tau-f_{\tau} \sin \tau\right) \frac{d \xi}{d s}+\left(f \sin \tau+f_{\tau} \cos \tau\right) \frac{d \eta}{d s}\right\} d s$,

in which the variable of integration has been changed into the Euclidean length of arc $s$. By the usual partial integration of the calculus of variations, one obtains

where

$$
\left[\begin{array}{l}
d l \\
d \alpha
\end{array}\right]_{a=0}=\int_{s_{0}}^{s_{1}}\{P \xi+Q \eta\} d s
$$

$$
P=f_{x}-\frac{d}{d s}\left(f \cos \tau-f_{\tau} \sin \tau\right), \quad Q=f_{y}-\frac{d}{d s}\left(f \sin \tau+f_{\tau} \cos \tau\right) .
$$

The integral (5) must be zero for all functions $\xi$ and $\eta$ vanishing at $t_{0}$ and $t_{1}$ and having continuous derivatives. Hence along the minimizing curve $C$ the two conditions

$$
P=0, \quad Q=0
$$

must be satisfied. These equations are, however, equivalent to the single equation

$$
f_{x} \sin \tau-f_{y} \cos \tau+f_{x \tau} \cos \tau+f_{y \tau} \sin \tau+\left(f+f_{\tau \tau}\right)_{l s}^{d \tau}=0 .
$$

For denote the left member of the last equation by $T$. Then the relations hold.

$$
P=T \sin \tau, \quad Q=-T \cos \tau,
$$

The differential equation (7) is the Euler equation * of the calculus of variations for the integral (2), and must be satisfied by every curve $C$ having continuous first and second derivatives which makes the integral I a minimum,

\footnotetext{
* Bolza, loc. cit., pp. 20, 123.
} 
that is, by the shortest lines in the geometry for which the integral (2) is defined to be the length integral.

In order to obtain the parametric representation of the extremal, the three equations

$$
\begin{aligned}
& \frac{d x}{d s}=\cos \tau, \\
& \frac{d y}{d s}=\cos \tau, \\
& \frac{d \tau}{d s}=\frac{f_{x} \sin \tau-f_{y} \cos \tau+f_{x \tau} \cos \tau+f_{y \tau} \sin \tau}{f+f_{\tau \tau}},
\end{aligned}
$$

must be integrated. From the properties assumed for the function $f$ and the known existence theorems for differential equations, ${ }^{*}$ the following theorem may be stated:

Through a given point $x_{0}, y_{0}$ and direction $\tau_{0}$ there passes one and but one shortest line (extremal) solving equation (7). Analytically these extremals have the form

$$
x=\phi\left(s, x_{0}, y_{0}, \tau_{0}\right), \quad y=\psi\left(s, x_{0}, y_{0}, \tau_{0}\right),
$$

where the initial values of $\phi$ and $\psi$ at $s=0$ are such that

$$
\begin{array}{ll}
x_{0} \equiv \phi\left(0, x_{0}, y_{0}, \tau_{0}\right), & \cos \tau_{0} \equiv \phi_{s}\left(0, x_{0}, y_{0}, \tau_{0}\right), \\
y_{0} \equiv \psi\left(0, x_{0}, y_{0}, \tau_{0}\right), & \sin \tau_{0} \equiv \psi_{0}\left(0, x_{0}, y_{0}, \tau_{0}\right) .
\end{array}
$$

As for the further properties of the functions $\phi$ and $\psi$, the existence theorems show that in a suitably chosen region the solutions $x, y, \tau$ of equations (8) must have first partial derivatives continuous in all arguments. From the first of these equations it follows easily that $\phi$ has also continuous derivatives $\phi_{e s}$ and $\phi_{s \tau_{0}}$, and similarly for the function $\psi$.

As the extremals correspond to straight lines in Euclidean geometry, so another class of curves called in the calculus of variations transversals, $\uparrow$ correspond to the Euclidean circles. Consider all the curves (9) which pass through a fixed point $O\left(x_{0}, y_{0}\right)$. On each of these mark off an arc such that the generalized length has a fixed value $r$. The value of $s$

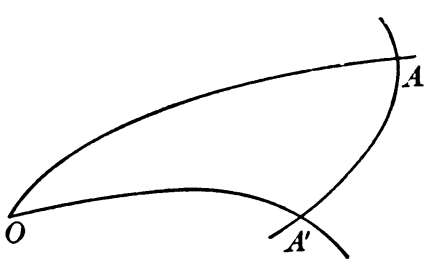

Fig. 1. determining the end-point $A$ of this arc (see Fig. 1) is defined by the equation

$$
r=\int_{0}^{s} f(\phi, \psi, \tau) d s
$$

* Buiss, Annsls of Mathematics, vol. 6 (1905), p. 49.

† Bolza, loc. cit., p. 167. 
Since the derivative for $s$ of the right member is $f\left(x_{0}, y_{0}, r_{0}\right)$ at $s=0$, and by hypothesis different from zero, this equation can be solved for $s$ in terms of $r$ and $\tau_{0}$, and the solution will have continuous first partial derivatives for both arguments in a properly chosen region. * Since

$$
\partial s=\frac{1}{f(\phi, \psi, \tau)}
$$

is greater than zero near the point $O$, the value of $s$ will increase from zero with $r$. The equation of the transversal $A^{\prime} A$ is found by substituting this value of $s$ in equations (9). The variables $x$ and $y$ then become functions of $\tau_{0}$ and $r$, and the points found by keeping $r$ constant and assigning different values to $\tau_{0}$ constitute the transversal $A^{\prime} A$ at the generalized distance $r$ from $O$.

The slope of the transversal at any point $A$ is not difficult to find. In order that the calculation may be analogous to that made above in deducing EULER's equation, equation (11) may be written

since

$$
r=\int_{0}^{s} f(\phi, \psi, \tau) \sqrt{\phi_{s}^{2}+} \overline{\psi_{s}^{2}} d s,
$$

$$
\phi_{s}^{2}+\psi_{s}^{2} \equiv 1
$$

Derivation for $\tau_{0}$ gives

$$
0=f(\phi, \psi, \tau) \frac{\partial s}{\partial \tau_{0}}+\int_{0}^{c} \frac{\partial}{\partial \tau_{0}}\left\{f(\phi, \psi, \tau) \sqrt{\phi_{s}^{2}+\psi_{s}^{2}}\right\} d s,
$$

or by the same process of partial integration used above in finding the extremals,

$$
0=f \frac{\partial s}{\partial \tau_{0}}+\left(f \cos \tau-f_{\tau} \sin \tau\right) \phi_{\tau_{0}}+\left(f \sin \tau+f_{\tau} \cos \tau\right) \psi_{\tau_{0}} .
$$

In deriving this result one must remember that the curves (9) are all extremals satisfying equations (6), so that the integral part of the expression disappears. Furthermore $\phi_{\tau_{0}}$ and $\psi_{\tau_{0}}$ vanish identically when $s=0$ on account of the initial conditions (10). The value of $d x / d \tau_{0}$, which with $d y / d \tau_{0}$ define the slope of the transversal, is found from the equation

$$
\frac{d x}{d \tau_{0}}=\cos \tau \frac{\partial s}{\partial \tau_{0}}+\phi_{\tau_{0}},
$$

where $\cos \tau$ is substituted in place of its equal $\phi_{.}$. By introducing the value of $\partial s / \partial \tau_{0}$ from (13) and using the relation (12), it follows that

$$
\frac{d x}{d \bar{\tau}_{0}}=\frac{\phi_{\tau_{0}} \sin \tau-\psi_{\tau_{0}} \cos \tau}{f}\left(f \sin \tau+f_{\tau} \cos \tau\right),
$$

\footnotetext{
* For the theorems on implicit functions, see Goursat, Cours d'Analyse, p. 40, or the translation of the same by HEDEICK, p. 35.
} 
and by a similar calculation,

$$
\frac{d y}{d \tau_{0}}=\frac{\phi_{\tau_{0}} \sin \tau-\psi_{\tau_{0}} \cos \tau}{f}\left(-f \cos \tau+f_{\tau} \sin \tau\right) .
$$

From these two equations it is seen that the direction of the transversal, being determined by the parentheses in the right members, depends only upcn the values of $x, y, \tau$ at the point $A$ and is independent of the center and radius of the generalized circle.

At any point $(x, y)$ on an extremal whose tangent at $(x, y)$ makes an angle $\tau$ with the $x$-axis, the direction defined by

$$
\cos \bar{\tau}=\frac{f \sin \tau+f_{\tau} \cos \tau}{\sqrt{f^{2}+f_{\tau}^{2}}}, \quad \sin \bar{\tau}=\frac{-f \cos \tau+f_{\tau} \sin \tau}{\sqrt{f^{2}+f_{\tau}^{\overline{2}}}},
$$

where the arguments of $f$ and $f_{\tau}$ are $x, y, \tau$, is the direction which a generalized circle (transversal) to the extremal through the point $(x, y)$ must have.* From the two equations (14) and (15) the parametric representation of the generalized circle may be found by integration in the form

$$
x=g\left(\tau_{0}, r\right), \quad y=h\left(\tau_{0}, r\right),
$$

where $\tau_{0}$ is the variable parameter, and $r$ the generalized distance of the circle from the center $O$.

For in the right hand members of (14) and (15), $\phi, \psi, \cos \tau=\phi_{s}$, and $\sin \tau=\psi$, are all functions of $s$ and $\tau_{0}$, and $s$ is to be expressed in terms of $r$ and $\tau_{0}$ from equation (11).

\section{§2. The notion of angle and its generalization.}

With the preceding results in mind the generalized angle corresponding to the integral (2) may be readily derived. In Euclidean geometry the angle between two straight line segments $O A^{\prime}$ and $O A$, is the ratio of the length $l$ of a circular arc $A^{\prime} A$ about $O$, divided by the radius $r$ of the circle. This measure of angle is independent of the particular circle chosen, however small its radius may be. In the geometries in which the generalization of length of $\S 1$ is presupposed, the angle between two extremals may be found in an analogous manner. The ratio $l / r$, in Euclidean geometry independent of the radius, is in general not so, and consequently a limit must be introduced.

If $O A^{\prime}$ and $O A$ (see Fig. 1) are two extremals through the point $O$, and $A^{\prime} A$ is the arc of length $l$ of a transversal at the generalized distance $r$ from $O$, then the generalized angle between $O A^{\prime}$ and $O A$ is defined to be the limit of the ratio $l / r$ as $r$ approaches zero.

* Compare with Bolza, loc. cit., pp. 36, 155, 197. 
Although the newly defined angle is a somewhat apparent extension of the usual notion, the calculation of its value as a definite integral is not so simple. Let $\bar{x}, \bar{y}, \bar{\tau}$ denote for the present the coördinates and direction of tangent at points on the transversal $A^{\prime} A$. It will be remembered that $\bar{x}$ and $\bar{y}$ are expressible from (14) and (15) in terms of $\tau_{0}$ and $r, \tau_{0}$ being the parameter and $r$ specifying the generalized distance of the transversal from $O$. If $\tau_{0}^{\prime}$ and $\tau_{0}$ are the initial directions of $O A^{\prime}$ and $O A$ at $O$, the generalized length of the arc $A^{\prime} A$ will be

$$
l=\int_{\tau_{0}^{\prime}}^{\tau_{0}} f(\bar{x}, \bar{y}, \bar{\tau}) \sqrt{\left(\frac{d \bar{x}}{d \tau_{0}}\right)^{2}+\left(\frac{d \bar{y}}{d \tau_{0}}\right)^{2}} d \tau_{0} .
$$

The integrand of this integral is a function of $\tau_{0}$ and $r$ having continuous first partial derivatives, and hence as $r$ approaches zero $l$ approaches as a limit the integral over the integrand taken for $r=0$. The limit of the ratio $l / r$ depends for its value upon the limit of the integrand in the preceding integral divided by $r$, which in turn is known when

$$
\lim \frac{1}{r} \frac{d x}{d \tau_{0}} \quad \text { and } \quad \lim \frac{1}{r} \frac{d y}{d \tau_{0}}
$$

have been calculated. From equations (14) and (15) it is seen that these last expressions are linear in $\phi_{\tau_{0}} / r$ and $\psi_{\tau_{0}} / r$, whose limits can be found in the manner explained in the following paragraph.

By TAYLOR's formula,

$$
\phi_{\tau_{0}}\left(s, \tau_{0}\right)=\phi_{\tau_{0}}\left(0, \tau_{0}\right)+s \phi_{s \tau_{0}}\left(\theta s, \tau_{0}\right) \quad(0<\theta<1),
$$

where the arguments $x_{0}, y_{0}$ of $\phi$, being considered fixed, are omitted. The first term of the right member is identically zero, as has been seen above. From equation (11)

$$
\frac{d s}{d r}=\frac{1}{f(\phi, \psi, \tau)},
$$

and therefore with the help of equations (10)

It follows that

$$
\lim _{r=0} \frac{s}{r}=\frac{1}{f\left(x_{0}, y_{0}, \tau_{0}\right)}
$$

$$
\lim _{r=0} \frac{\phi_{\tau_{0}}\left(s, \tau_{0}\right)}{r}=\frac{\phi_{e \tau_{0}}\left(0, \tau_{0}\right)}{f\left(x_{0}, y_{0}, \tau_{0}\right)}=-\frac{\sin \tau_{0}}{f\left(x_{0}, y_{0}, \tau_{0}\right)},
$$

the last step because, from (10),

$$
-\sin \tau_{0} \equiv \phi_{s \tau_{0}}\left(0, \tau_{0}\right)
$$

By a similar process of reasoning,

$$
\lim \frac{\Psi_{\tau_{0}}(s, r)}{r}=\frac{\cos \tau_{0}}{f\left(x_{0}, y_{0}, \tau_{0}\right)}
$$


From equations (14) and (15),

$$
\begin{aligned}
& \lim _{r=0} \frac{1}{r} \frac{d x}{d \tau_{0}}=-\frac{f \sin \tau_{0}+f_{\tau} \cos \tau_{0}}{f^{2}}, \\
& \lim _{r=0} \frac{1}{r} \frac{d y}{d \tau_{0}}=\frac{f \cos \tau_{0}-f_{\tau} \sin \tau_{0}}{f^{2}},
\end{aligned}
$$

where the arguments of $f$ and $f_{\tau}$ are $x_{0}, y_{0}, \tau_{0}$. The limit of $l / r$ follows from equations (16) and (17).

The generalized angle at a point $(x, y)$ between two directions whose Euclidean angles with the $x$-axis are $\tau^{\prime}$ and $\tau$, has therefore the value

$$
\lim _{r \doteq 0} \frac{l}{r}=\int_{\tau^{\prime}}^{\tau} \frac{f(x, y, \bar{\tau})}{[f(x, y, \tau)]^{2}} \frac{f^{2}+f_{\tau}^{\bar{z}}}{[x} d \tau,
$$

where the arguments of $f$ and $f_{\tau}$ are $x, y, \tau$, and $\bar{\tau}$ is defined by the equations

$$
\begin{aligned}
& \cos \bar{\tau}=\frac{f \sin \tau+f_{\tau} \cos \tau}{\sqrt{f^{2}+f_{\tau}^{2}},} \\
& \sin \bar{\tau}=\frac{-f \cos \tau+f_{\tau} \sin \tau}{v^{\prime} \bar{f}^{2}+f_{\tau}^{2}},
\end{aligned}
$$

i. e., $\bar{\tau}$ is the direction transverse to $\tau$ at the point $O(x, y)$.

For simplicity the subscript zero designating the point $O$ has been dropped. The integral (18) might be written somewhat more simply

$$
\int_{\tau^{\prime}}^{\tau} f(x, y, y, \bar{\tau}) \csc (\tau-\bar{\tau}) d \tau .
$$

\section{§ 3. Application to special cases.}

As a first application one would naturally be interested to see the behavior of the integral (18) in the case of ordinary Euclidean geometry. The function $f(x, y, \tau)$ is then simply unity, and the generalized angle becomes $\tau-\tau^{\prime}$ as would be expected.

The expression for the length of a curve on a surface when the surface is given in parametric representation, is an integral (2) in which the function $f$ has the value

$$
f(x, y, \tau)=\sqrt{ } E^{\cos ^{2} \tau+2 F} \overline{\cos \tau \sin \tau+G \sin ^{2} \tau} .^{*}
$$

To conform to the previous notations of this paper, $x$ and $y$ are used as the arguments of $E, F$, and $G$ instead of the customary $u, v$. As is well known the

* See for example GAUss, General Investigations of Curved Surfaces (translation), p. 20, or his Werke, vol. 4, p. 236. 
angle $\omega$ between any curve and a line $y=$ const. on the surface, is defined by the formulæ*

$$
\begin{aligned}
& \cos \omega=\frac{E \cos \tau+F \sin \tau}{\sqrt{E} \sqrt{E \cos ^{2} \tau+2 F \cos \tau \sin \tau+G \sin ^{2} \tau},} \\
& \sin \omega=\frac{\sqrt{E G-F^{2}} \cdot \sin \tau}{\sqrt{E \sqrt{E \cos ^{2} \tau+2 F \cos \tau \sin \tau+G \sin ^{2} \tau}},}
\end{aligned}
$$

where $\tau$ now represents the angle between the $x$-axis and the tangent to the image of the curve in the $x y$-plane.

The generalized angle defined by the integral (18) when $f(x, y, \tau)$ has the form (20), is exactly the same as the ordinary angle $\omega$ calculated from the formula (21) between two curves on the surface whose images in the xy-plane make angles of $\tau^{\prime}$ and $\tau$ with the $x$-axis.

In order to prove this it is only necessary to find $d \omega / d \tau$ and show that it has a value identical with that of the integrand of the integral (18) when the value of $f(x, y, \tau)$ from (20) is substituted. The calculation is simplified by the introduction of the notations

Then

$$
\lambda=E \cos \tau+F \sin \tau, \quad \mu=F \cos \tau+G \sin \tau .
$$

$$
f=\frac{\lambda \cos \tau+\mu \sin \tau}{f}, \quad f_{\tau}=\frac{-\lambda \sin \tau+\mu \cos \tau}{f},
$$

and from formulæ (19)

$$
\cos \bar{\tau}=\frac{\lambda}{\sqrt{\lambda^{2}+\mu^{2}}}, \quad \sin \bar{\tau}=\frac{-\mu}{\sqrt{\lambda^{2}+\mu^{2}}} .
$$

The values of $f(x, y, \bar{\tau})$ and $f^{2}+f_{\tau}^{2}$ are

$$
f(x, y, \bar{\tau})=\sqrt{E G-F^{2}} \frac{f}{\sqrt{\lambda^{2}+\mu^{2}}}, \quad f^{2}+f_{\tau}^{2}=\frac{\lambda^{2}+\mu^{2}}{f^{2}},
$$

so that the final value of the integrand of $(18)$ is

$$
\frac{f(x, y, \bar{\tau})}{f(x, y, \tau)} \csc (\tau-\bar{\tau})=\frac{\sqrt{E} \overline{G-F^{2}}}{E \cos ^{2} \tau+\overline{2} F \cos \tau \sin \tau+G \overline{\sin ^{2}} \bar{\tau}},
$$

But this is the value of $d \omega / d \tau$, easily calculable from equations (21).

The non-Euclidean geometries in the plane correspond, as is well known, to the geometries on surfaces of constant positive and negative curvature. By a projective transformation of the plane, or of the parameters of the surface according to the point of view which one wishes to take, the length integral can be transformed so that the function $f(x, y, \tau)$ has one of the two forms $\dagger$

\footnotetext{
* See Gauss, Translation, p. 26 ; Werke, vol. 4, p. 242.

† Darboux, Theorie générale des Surfaces, vol. 3, p. 62; KLEIN, Nichteuklidische Geometrie,
} p. 121. 


$$
f(x, y, \tau)=\frac{\sqrt{\left(a^{2} \pm y^{2}\right) \cos ^{2} \tau \mp 2 x y \cos \tau \sin \tau+\left(a^{2} \pm x^{2}\right) \sin ^{2} \tau}}{x^{2}+y^{2} \pm a^{2}}
$$

the upper signs corresponding to elliptic, the lower to hyperbolic geometry. Only the hyperbolic geometry will be considered here on account of the close analogy between the calculations in the two cases.

The elements of the hyperbolic geometry connected with the function (23) are the points interior to the circle

$$
x^{2}+y^{2}=a^{2} .
$$

It is usual to define the length of a straight line segment $O A$ as a multiple of the logarithm of the anharmonic ratio of the points $O$ and $A$ with the two intersections $B_{1}$ and $B_{2}$ of the line $O A$ and the circle (24).* It can be shown, how ever, that this definition is identical with the one given above in the form of a definite integral. For denote the coördinates of the point $O$ by $\left(x_{0}, y_{0}\right)$, of $A$ by $(x, y)$, and let $\tau$ be the Euclidean angle which the line $O A$ makes with the $x$-axis. If $s, s_{1}$ and $s_{2}$ are the Euclidean distances on the line from the point $O$ to $A, B_{1}$ and $B_{2}$ respec-

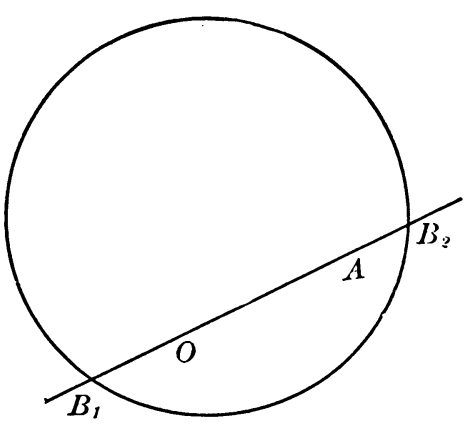

Fig. 2. tively, then the hyperbolic length of $O A$ according to the geometrical definition is

$$
l=\frac{1}{2} \log \left(\frac{s-s_{1}}{s-s_{2}} \cdot \frac{s_{2}}{s_{1}}\right) .
$$

The derivative of this expression for $s$ is

$$
\overline{d s}=\frac{1}{2} \frac{s_{1}-s_{2}}{\left(s-s_{1}\right)\left(s-s_{2}\right)},
$$

which must be equal to the function $f(x, y, \tau)$ defined by equation (23) if the analytical and geometrical definitions of length are to agree in the case of the straight line. The values of $\sigma_{1}=s_{1}-s$ and $\sigma_{2}=s_{2}-s$ are easily found by substituting $x+\sigma \cos \tau$ and $y+\sigma \sin \tau$ for $x$ and $y$ in equation (24) and solving for $\sigma$. It follows that

$$
\begin{aligned}
\sigma_{1} \sigma_{2} & =\left(s-s_{1}\right)\left(s-s_{2}\right)=x^{2}+y^{2}-a^{2}, \\
\sigma_{1}-\sigma_{2} & =s_{1}-s_{2}=2 \sqrt{\left(a^{2}-y^{2}\right) \cos ^{2} \tau+2 x y \cos \tau \sin \tau+\left(a^{2}-x^{2}\right) \sin ^{2} \tau,}
\end{aligned}
$$

which proves the equality of $d l / d s$ and $f(x, y, \tau)$.

In a similar manner it can be shown that the usual geometrical definition of

* KLEIN, Nichteuklidische Geometrie, p. 96. 
angle in hyperbolic geometry* gives the same value which one finds from the

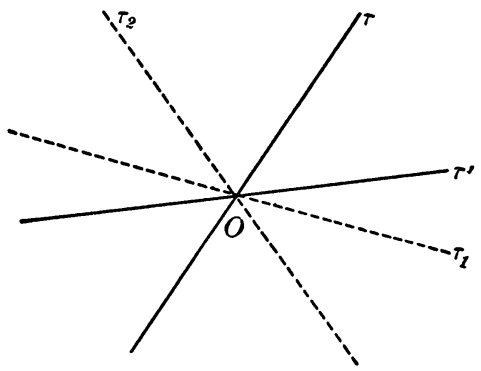

FIG. 3. expression (18). If $m_{1}$ and $m_{2}$ are the slopes of the two imaginary tangents (dotted in the figure) to the circle (24) through the point $O(x, y)$ interior to the circle, the hyperbolic angle $\omega$ between any other two directions making Euclidean angles $\tau^{\prime}$ and $\tau$ with the $x$-axis, is defined geometrically to be a multiple of the logarithm of the anharmonic ratio of the four directions whose tangents are $m_{1}=\tan \tau_{1}$, $m_{2}=\tan \tau_{2}, m^{\prime}=\tan \tau^{\prime}$, and $m=\tan \tau ;$ i. e.,

The derivative of this for $\tau$ is

$$
\omega=\frac{1}{2} \log \left(\frac{m-m_{1}}{m-m_{2}} \cdot \frac{m^{\prime}-m_{2}}{m^{\prime}-m_{1}}\right) \text {. }
$$

$$
\frac{d \omega}{d \tau}=\frac{1}{2} \frac{m_{1}-m_{2}}{\left(m-m_{1}\right)\left(m-m_{2}\right)} \sec ^{2} \tau .
$$

But $m_{1}$ and $m_{2}$ are the roots of the quadratic equation

$$
m^{2}\left(a^{2}-x^{2}\right)+2 m x y+\left(a^{2}-y^{2}\right)=0,
$$

and by substituting one finds

$$
\frac{d \omega}{d \tau}=\frac{a \sqrt{a^{2}-x^{2}-y^{2}}}{\left(a^{2}-y^{2}\right) \cos ^{2} \tau+2 x y \cos \tau \sin \tau+\left(a^{2}-x^{2}\right) \sin ^{2} \tau} .
$$

On the other hand, by comparison of equations (20) and (23),

$$
E=\frac{a^{2}-y^{2}}{\left(a^{2}-x^{2}-y^{2}\right)^{2}}, \quad F=\frac{2 x y}{\left(a^{2}-x^{2}-y^{2}\right)^{2}}, \quad G=\frac{a^{2}-x^{2}}{\left(a^{2}-x^{2}-y^{2}\right)^{2}},
$$

and from (22)

$$
\frac{f(x, y, \bar{\tau})}{f(x, y, \tau)} \csc (\tau-\bar{\tau})=\frac{a \sqrt{a^{2}-x^{2}-y^{2}}}{\left(a^{2}-y^{2}\right) \cos ^{2} \tau+2 x y \cos \tau \sin \tau+\left(a^{2}-x^{2}\right) \sin ^{2} \tau} .
$$

The expression (25), with $x, y, \tau$ considered fixed and $\tau$ variable, is therefore the primitive of the integral (18).

In the non-Euclidean hyperbolic and elliptic geometries, the geometrical definition of angle as a multiple of the logarithm of the anharmonic ratio of the lines bounding the angle taken with the two tangents to the fundamental conic through their point of intersection, is the same as the analytical definition by means of the definite integral (18).

* KLEIN, loc. cit., p. 87. 
In conclusion it might be interesting to indicate a possible generalization of a theorem of Gauss * concerning geodesic triangles. The theorem expresses the excess of the sum of the angles of a geodesic triangle on a surface over two right angles as the double integral of the curvature, multiplied by the element of area, of the surface taken over the same triangle. From this relation one easily derives that the sum of the angles of a triangle is greater than two right angles in elliptic, and less than two right angles in hyperbolic geometry, for these correspond to geometries on surfaces of constant positive and negative curvature respectively. For any problem of the calculus of variations there is an invariant connected with the second variation, more specifically with $\mathrm{J}_{\text {ACOBI's differential }}$ equation, which in the problem of geodesic lines reduces to the curvature of the surface. $\dagger$ It seems certain that a relation similar to GAUss's must exist between the generalized angles, as defined above, of a triangle whose sides are extremals, and the invariant just mentioned.

The University of Chicago, August, 1905.

* Translation, p. 30 ; Werke, vol. 4, p. 246.

† Darboux, loc. cit., p. 94 ff. 